\title{
Neurologische Erkrankungen - ein Update
}

$\mathrm{D}$ as Fach Neurologie ist in den letzten Jahren und Jahrzehnten erheblich in Bewegung geraten. Aus einem Teilbereich der Medizin, der den Ruf einer eher kontemplativen Disziplin hatte, hat sich ein Fach entwickelt, in dem bei zahlreichen Krankheiten rasche Diagnosen und Therapien möglich und erforderlich sind. Dabei hat die Neurologie im diagnostischen Bereich zunächst von der Fortentwicklung der klinischen Neurophysiologie und dann vor allem der Neuroradiologie profitiert. In der Akuttherapie sind Fortschritte insbesondere bei der Behandlung des Schlaganfalls und in der neurologischen Intensivmedizin erzielt worden.

In dieser Ausgabe des klinikarzt geben Philipps und Busse (Neurologische Klinik, Klinikum Minden) instruktive Antworten auf eine Reihe aktueller Fragen zum Thema Schlaganfall. Die Einführung der Thrombolyse, die Verbesserung der intensivmedizinischen Behandlungsstrategien, insbesondere aber der Aufbau zahlreicher Stroke Units haben sich für die Schlaganfallpatienten in Deutschland bezahlt gemacht. Eine bessere Aufklärung der Patienten und eine Verkürzung der Transportzeiten in die Klinik könnten noch wesentlich dazu beitragen, dass noch mehr Patienten von Maßnahmen wie der Thrombolyse profitieren. Für die Zukunft erhoffen wir uns unter anderem neuroprotektive Therapiestrategien, die minderperfundiertes zerebrales Gewebe schützen und das Zeitfenster für die Thrombolyse verlängern können. Ganz besonders wichtig sind beim Schlaganfall einschließlich der Hirnblutungen aber die Fortschritte in der Primär- und Sekundärprophylaxe, die sich vor allem durch eine Modifikation der Risikofaktoren - und hier in erster Linie der arteriellen Hypertonie (allgemein akzeptierter Zielwert nach Schlaganfall: maximal 130/85 mmHg!) - erreichen lassen.

Die Mehrzahl der neurologischen Erkrankungen verläuft chronisch und viele dieser Krankheitsbilder gehören nosologisch immer noch in den großen Topf der so genannten neurodegenerativen Erkrankungen - ihre Ätiologie ist also bislang noch nicht bekannt. Als eine der wichtigsten Krankheiten in diesem Zusammenhang ist der Morbus Parkinson zu nennen. Immer mehr erfahren wir heute über die Pathogenese dieser Erkrankung - ein Stichwort ist zum Beispiel das Ubiquitin-Proteasom-System. Trotzdem steht der Durchbruch, was die Ursache angeht, noch aus. Andererseits haben die letzten Jahre eine Verfeinerung der individuellen medikamentösen Therapiemöglichkeiten gebracht, und insbesondere mit der Implantation von Stimulatoren bieten sich heute deutlich verbesserte Behandlungsoptionen in den späten Phasen der Erkrankung an. Der Beitrag von CeballosBaumann (Neurologische Klinik der TU München) gibt hierzu einen aktuellen Überblick, der auch derzeit in Erprobung befindliche Therapien (z.B. die Implantation retinaler dopaminproduzierender Zellen) mit einbezieht.

Auch bei der Multiplen Sklerose (MS) sind die Ursachen weiterhin unklar, wenngleich fraglos autoimmunologische Prozesse eine wichtige pathogenetische Rolle spielen. Die Einführung immunmodulatorischer Therapien, vor allem der Beta-Interferone und des Glatirameracetats, und die Möglichkeit zur Eskalation mittels Mitoxantron bei progredientem Krankheitsverlauf haben die therapeutischen Aussichten bei der Multiplen Sklerose deutlich verbessert. Zudem erlauben neuere Diagnosekriterien unter Hinzuziehung des MRTs inzwischen, die Diagnose früher zu stellen und damit auch die Therapie früher einzuleiten. Derzeit werden Kombinationstherapien - etwa mit dem altbekannten Azathioprin - untersucht, von denen man sich weitere Verbesserungen erwartet. Kleinschnitz und Rieckmann (Neurologische Universitätsklinik Würzburg) fassen den aktuellen Stand bei Diagnostik und Therapie dieser wichtigen neurologischen Erkrankung knapp und verständlich zusammen.

Die Epilepsien sind das vierte neurologische Thema, dem sich diese Ausgabe des klinikarzt widmet. Auch diese Krankheitsgruppe besitzt, wie die zuvor angesprochenen Thematiken, große medizinische und volkswirtschaftliche Bedeutung. In den letzten Jahren wurde eine Reihe neuer Antiepileptika zugelassen. Steinhoff (Epilepsie-Zentrum Kehl-Kork) und Wildemann (Neurologische Klinik der Universität Heidelberg) heben in ihrem Beitrag hervor, dass diese die individuelle Behandlung der einzelnen Patienten erleichtern und die Nebenwirkungen reduzieren. Den Anteil pharmakoresistenter Epilepsien jedoch konnten auch diese Substanzen kaum verringern. Die Autoren betonen in ihrem instruktiven Beitrag die Bedeutung der Epilepsiechirurgie für diese pharmakoresistenten Epilepsien und verweisen speziell für die Temporallappenchirurgie auf günstige Studienergebnisse.

Insgesamt ergeben sich in der Neurologie auf vielen Teilgebieten diagnostische und therapeutische Fortschritte, die es selbst dem neurologischen Generalisten schwierig machen, immer auf dem neuesten Stand zu bleiben. Die vier Beiträge in diesem Heft, alle von namhaften Autoren auf ihrem Gebiet, sind eine gute Gelegenheit, sich einen kompetenten Überblick über die jeweiligen Gebiete zu verschaffen.
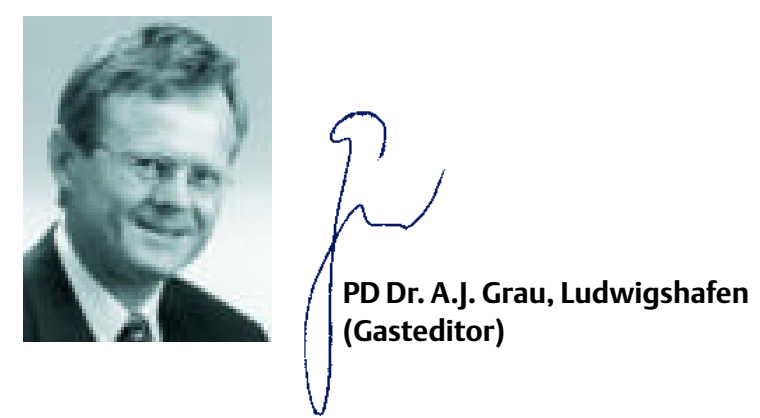

klinikarzt 2004; 33 (4) 